to incur the charge from their comrades of being malingerers for going into hospital with such trifling ailments; but this was a fault on the right side. Simple excoriations were quickly got rid of ; and chancres, being in their very first stage, rapidly healed under the local application of caustic and water dressing. The men were discharged to barracks in a few days; they had no extra duty to bring up, if they had not concealed their complaints; and their constitutions were saved from the poisoning of the syphilitic virus, or of mercury given for its cure. After some time, the old cases of secondary syphilis, and sloughing buboes and chancres, were got rid of ; and by the end of three months we had scarcely a case of syphilitic disease in the hospital.

The health-inspections took place, as usual, once a week. The men were paraded in their several barrack-rooms, each man, in cold weather, standing on his stockings; legs and feet bare; sleeves and trousers turned up; jacket and shirt-collar thrown open, to expose the throat and chest; hands, face, feet, and legs, washed clean. In passing down the line, it could be seen at a glace if the men had the appearance of health, and were free from ophthalmia, itch, ulcers of the legs, etc.; but no examination was made for venereal disease, because I felt satisfied that such cases would be honestly reported. I therefore left it to the soldier's own honour and sense of duty, as well as self-interest, to come forward themselves; and I must do them the justice to say, I seldom-I believe I may safely add, never-had reason to regret the confidence I placed in them; while I know that they considered the exemption from personal examination in public too great a boon and indulgence to be lightly thrown away.

\section{CASE OF EPILEPSY.}

By Augtustin Prichard, Esq., Surgeon, Clifton.

THE interesting report of the last illness and post mortem ex. amination of the late Professor Alison of Edinburgh, which is published in the Edinburgh Monthly Journal for January of this year, reminded me so strongly of a case which was under my care some years ago, that I think it worthy of record.

I obtained the following account of the early life and the medical history of my patient from his cousin, a late practitioner of this city, who had known him well, and from his wife, who survived him; but I had myself known him for some years before his death, and he had been under my medical care.

B. R. died in his seventy.first year, having been insensible from apoplexy for two days before. He had been totally blind from amaurosis, and nearly deaf for fifty-seven years, and had been the subject of epilepsy since his fourteenth year; that is, for about the same time. He had never less than three or four fits daily during this long period, and sometimes he had between twenty and thirty. He made baskets for his livelihood; and, when he felt a fit coming on, he would lay aside his work, and seat himself upon the floor with his back against the wall, and in this sitting posture he would remain until the fit had passed by; and when he recovered himself, he would return to his work. He and his wife were so accustomed to this proceeding, that they thought nothing of it, until two days before his death, when his wife found that he remained insensible, and as it turned out, his epilepsy had changed into a fatal apoplexy.

Taking into calculation the lowest daily number of fits which this poor fellow had, the entire number must have amounted to at least sixty thousand, and probably was very many more ; and, notwithstanding this almost incredible series of attacks in his brain, his intellects, such as they were, considering that their development had been checked by the early loss of the two most important senses, sight and hearing, were unimpaired and unaffected, and he lived until he had completed the term usually described as the age of man.

Post Mortem Examination. The head only was examined. The dura mater externally was natural, but the arachnoid was very opaque. The ventricles of the brain were filled with fluid and coagula, being the cause of death. In the falx major anteriorly, but not quite touching the crista galli of the ethmoid, was a considerable plate of bone, an inch and a half long, tolerably thick, flat upon one side where it appeared to be rather lying upon or adherent to the fibrous membrane, than in its substance, and upon the other side it was marked by the contiguous convolutions of the brain as distinctly as we see them upon the orbital plate of the frontal bone. At the upper part of the convexity of the left hemisphere were two round deposits of bone, as large as nuts, under the arachnoid and in the pia mater, pressing down into the substance of the brain, which was much softened around them. There was a single, much larger deposit of the same shape below, and another was attached to the upper surface of the petrous portion of the temporal bone by a pedicle, and occupied a corresponding cavity in the substance of the middle lobe.

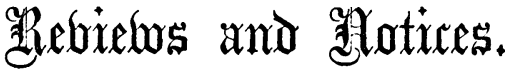

Chintcal Lectures on Certain Acute Diseases. By Robert Bentley Todd, M.D., F.R.S. Pp. 48\%. London: John Churchill. I860.

To no physician of this age is medicine more indebted than to the late Dr. Tonn. To him more than to any other is due the credit of having directed the attention of the profession to two cardinal points in therapeutics-the immense value, in certain conditions of the system, of direct and unsparing stimulation; and, secondly, the uselessness of quantitative treatment (depletion) in local inflammations dependent on qualitative bloodderangement. We would not by any means constitute ourselves the indiscriminate advocates of Dr. Todd's practice, nor affirm that in carrying out these dogmata his treatment of disease may not sometimes have been faulty and open to criticism; but we believe his errors, where he erred, to have been errors of detail, not of principle; and the element of truth, involved in the two propositions we have referred to, is so great and of such vast practical import, that by the side of them any error in their application is forgotten. That many diseased conditions which tradition has told us are hyperdynamic are really adynamic; that disease is not merely vital power run riot, but vital power depressed, not merely divergent, but enfeebled physiology; that for a restoration from disease to health we must fall back on the same powers that maintain a state of health; that idiopathic inflammation, if there is such a thing, is extremely rare; that local inflammation is in almost every instance, unless surgical, demonstrably dependent on some special blood-contamination-these were the doctrines Dr. Todd was always enforcing; on which he based his treatment; and on which the volume before us contains his latest convictions.

This volume is the third, and now the last, of a series of Clinical Lectures on various subjects, taken in accordance with the materials for clinical study furnished by the wards of King's College Hospital, and reported successively by $\mathrm{Mr}$. James Salter, Dr. Hyde Salter, Dr. Lionel Beale, and Dr. Conway Evans. The three volumes taken together constitute a valuable mass of practical instruction, and fairly represent the author's clinical teaching for the last twelve years of his life.

The number of specific subjects treated of is small: the lectures are distinctly stated to be "on certain acute diseases"; but the principles laid down in them are of universal application, and, as far as therapeutics go, they may be said to be on acute disease in general. The first three lectures are on Rheu. matic Fever; the fourth and fifth on Continued Fever; the sixth on Erysipelas; the seventh on a so-called Erysipelatous Inflammation of the Fauces; four lectures are on Pneumonia; one on Pyæmia; one on the Treatment of Acute Internal Inflammations generally; and the last on the Therapeutical Influence of Alcohol. These last two are perhaps the most interesting of all, involving as they do the direct discussion of those principles on which Dr. Todd based so much of his therapeutics.

In his preface, which is as valuable a part of the work as any, Dr. Todd thus sums up the conclusions which the clinical observations detailed in the lectures tend more or less to establish :-

"1. That the notion so long prevalent in the schools, that 